\title{
The interdependence of pitch and temporal judgments by absolute pitch possessors
}

\author{
SUMI SHIGENO \\ Kitasato University, Kitasato, Sagamihara-shi, Kanagawa, Japan
}

\begin{abstract}
The auditory tau and the kappa effects show that there is time-pitch interdependence in our perception. Our judgments of pitch separation between two tones depend on the temporal interval between them (the auditory tau effect), and our judgments of the tones' temporal interval depend on their pitch separation (the kappa effect). The mechanisms underlying this interdependence were investigated by studying the auditory tau and the kappa effect in three experiments. Comparisons were made between results obtained from subjects with absolute pitch and those who did not have absolute pitch, and two frequency ranges of pure tones (octave and whole-tone conditions) were selected. The procedures had been used in previous experiments (Shigeno, 1986), in which the auditory tau and the kappa effects were compared in speech and nonspeech stimuli. The present results demonstrate that the auditory tau effect does not occur when possessors of absolute pitch judge the closeness of stimuli in pitch, except when the stimulus continuum consists of tones that do not correspond to musical notes in the whole-tone condition. The kappa effect was obtained in the judgment of possessors of absolute pitch in both the octave and the wholetone conditions. These findings suggest that the interaction between temporal interval and pitch judgment might be explained in terms of the two different memory modes for retaining the pitch of tones, and that these effects occur at the precategorical level.
\end{abstract}

The relationship between temporal and spatial perception has been of interest in psychology. The tau and kappa effects are usually cited as evidence for the dependence of spatial perception on temporal separation and for the dependence of temporal perception on spatial distance, respectively. The tau effect was first identified by Helson (1930) in tactile perception. He found that when two points are stimulated successively, the perceived spatial distance between them varies as a function of the temporal interval between the stimulations. Thus, if three points are marked off on an observer's skin and the temporal interval between the stimulation of the first and second points is greater than that between the second and third, the observer reports that the spatial distance between the first and second points is greater than that between the second and third, even though, in fact, it may be physically equal or less.

Cohen, Hansel, and Sylvester (1954), using the method of adjustment, found an effect similar to the tau effect in bisection judgments of pitch and labeled it the auditory tau effect. In the auditory tau experiment, a subject hears a succession of three different tones of brief and equal duration-but with unequal temporal spacing-and is asked to adjust the middle tone to appear to be intermediate in pitch between the other two. When the middle tone is temporally close to either of the two neighboring tones,

This research was supported in part by Grant-in-Aid for Scientific Research 02610064 from the Ministry of Education, Science, and Culture. Correspondence should be addressed to S. Shigeno, College of Liberal Arts and Sciences, Kitasato University, 1-15-1 Kitasato, Sagamihara-shi, Kanagawa, 228 Japan. it is adjusted farther apart in frequency than when it is more temporally distant. In other words, the subject, having perceived the middle tone to be nearer in pitch to the temporally closer tone, adjusts the middle tone as being different in frequency from its actual frequency. In this case, "pitch"' is considered to be equivalent to "space," and the separation in frequency between two tones may correspond to the auditory "distance."

Meanwhile, other research on the relationship of temporal and spatial perception has focused on the effects of variations in visual space on perceived temporal durations. Abbe (1936) showed that when two equal temporal intervals are separated by stimuli demarcating different spatial extents, stimuli with a larger spatial extent result in a longer perceived duration of the temporal interval. Similar results have been demonstrated by Price-Williams (1954) and by Cohen, Hansel, and Sylvester (1953), who labeled their findings the kappa effect, to distinguish them from the tau effect. The kappa effect has been regarded as the phenomenal converse of the tau effect. The investigations of the tau and kappa effects suggest that there is a tradeoff between temporal and spatial perception. However, the mechanisms underlying this interdependence of temporal and spatial perception remain unexplained. In most of the previous experiments on this topic, the method of adjustment was used, and the mechanisms of the tau and kappa effects have been explained by factors that greatly depend on this experimental procedure (see, e.g., Christensen \& Huang, 1979).

Shigeno (1986), using similarity judgments in an AXB paradigm, found an effect similar to the auditory tau effect (i.e., assimilation to the temporally closer sound) not 
only in the judgment of the pitch of pure tones, but also in the judgment of vowels of stimulus continua that consisted of "within-phonetic-category" stimuli $(/ \mathrm{i} /-/ \mathrm{I} /$ and $/ \mathrm{I} /-/ \mathrm{i} /$ continua). A pattern opposite to that of the auditory tau effect (i.e., contrast to the temporally closer sound) was also obtained in the judgment of stimulus continua that consisted of "between-phonetic-category" stimuli (/i/-/e/ and /e/-/i/ continua). The pitch and vowel judgments are influenced by the temporal position of a previous or trailing stimulus in one stimulus set.

Shigeno (1986) found further that the kappa effect occurs for both synthetic vowels (speech stimuli) and pure tones (nonspeech stimuli). Although the magnitude of context effects that are a function of temporal separation between two stimuli (the auditory tau effect) greatly depends on stimulus attributes, the magnitude of context effects that are a function of spatial separation on the stimulus continuum between two stimuli (the kappa effect) is scarcely influenced by stimulus attributes (e.g., speech vs. nonspeech) or by judgmental modes (e.g., phonetic vs. nonphonetic). Shigeno explained these results on the basis of the model proposed by Shigeno and Fujisaki (1979) and suggested that the results from the experiment on the auditory tau effect indicated that the hierarchical auditory information processing is influenced by temporal separation between two stimuli at two different perceptual levels: the auditory (precategorical) and the phonetic (categorical). The results from the experiment on the kappa effect, on the other hand, show that temporal perception is influenced by spatial separation on the stimulus continuum (separation in frequency) between two stimuli at the nonphonetic (precategorical) level, but not at the phonetic (categorical) level. Shigeno thus concluded that the interaction between temporal interval and pitch (or vowel) judgments may be explained on the basis of the integration of forward and backward context effects, and suggested further that, on the basis of the results from the use of speech and nonspeech stimuli, the interaction between temporal and spatial judgments is not symmetrical and occurs at an early stage of auditory information processing.

Some common characteristics have been found in the perception of both speech and nonspeech stimuli. For example, categorical perception can be observed in the perception of musical intervals (Burns \& Ward, 1978; Siegel \& Siegel, 1977a), in the perception of a rise-time continuum (Cutting \& Rosner, 1974), in the perception of the relative onset time of two component tones (Pisoni, 1977), and in the perception of the duration of rapid spectrum changes (Pisoni, Carrell, \& Gans, 1983), as well as in the perception of speech sounds. Siegel and Siegel (1977a) suggested that musicians acquire categories for pitch that are functionally similar to phonemic categories for speech. Burns and Ward (1978) found that when procedures equivalent to those typically used in speechperception experiments were employed (i.e., determination of identification and discrimination functions for stimuli separated by equal physical increments), musical intervals were perceived categorically by trained musicians, although the results of other experiments imply that this dependence of categorical perception on procedure may be related to differences in stimulus uncertainty between the procedures. They concluded that (1) speech-specific models obviously are not applicable; (2) there is no evidence that musical-interval discriminations are based on information from more than one processing stage as information-processing-type models propose; and (3) there is no evidence for the existence of natural perceptual boundaries of melodic musical intervals, although such boundaries clearly exist in certain speech continua. Categorical perception of nonspeech stimuli argues against the notion that the perceptual mechanisms of speech are specific among the auditory modalities.

People with absolute pitch are able to identify or produce musical pitch without reference to any standard tones, just as they identify and produce speech sounds. In this sense, their judgments are absolute. With a reference tone, even those who lack absolute pitch yet have some musical experience can identify the name of a target tone rather accurately, mainly on the basis of a musical context. That is, their judgments are made on the basis of musical interval sense (relative pitch).

Another example of characteristics shared by speech and nonspeech stimuli may be that there seem to be critical ages in the acquisition of language and absolute pitch. It has been debated whether absolute pitch is innate or acquired as a result of specific learning experiences at an early age (Ward \& Burns, 1982). Some people can acquire absolute pitch if they are specifically trained to categorize tones into musical notes when they are very young, usually between 4 and 7 years of age (Radocy \& Boyle, 1979). This problem, however, is still at issue. Similarly, ordinary people can become bilingual if they have had some experience with a foreign language in childhood. It is almost impossible to acquire the ability to discriminate (or pronounce and perceive correctly) the different phonemes of a new language after adolescence (Borden \& Harris, 1984).

The present study is an attempt to answer certain questions about the interdependence of auditory and temporal judgments, with especial attention to the perceptual processes of those who have absolute pitch. Three experiments were conducted to investigate the judgments of people with absolute pitch regarding the closeness of stimuli in pitch or in time, mainly from the view of the time-pitch interaction-the auditory tau and kappa effects-and to examine the perceptual processes of possessors of absolute pitch. Experiments 1 and 2 addressed the auditory tau effect, and Experiment 3, the kappa effect. Comparisons were made between subjects with absolute pitch and subjects without it.

\section{EXPERIMENT 1}

The first purpose of Experiment 1 was to investigate whether the auditory tau effect (or the reverse pattern of the auditory tau effect) could be obtained in pitch judgments made by people with absolute pitch. The second purpose was to examine whether or not people with ab- 
solute pitch perceive tones by means of a process that is analogous to speech perception. The procedure employed in Experiment 1 was similar to that used in the auditory tau effect experiment on speech perception carried out by Shigeno (1986). Shigeno employed an AXB paradigm to elicit similarity ratings that were subsequently used to examine the auditory tau effect in the pitch judgment of subjects who did not have absolute pitch. According to this paradigm, subjects hear one triad and judge whether the second stimulus $(\mathrm{X})$ is similar to the first or to the third. Since the auditory tau effect in pitch bisection judgments obtained with the method of adjustment might be based on the interaction of memories of more than three stimuli (Christensen \& Huang, 1979), an AXB paradigm was employed here to induce performance that would more directly reflect the effect of temporal variables on judgments of closeness in pitch between stimuli.

\section{Method}

Subjects. The subjects were separated into two groups. One consisted of subjects with absolute pitch (AP group); the other, of subjects without absolute pitch (non-AP group). Prior to the experiment, subjects were tested to determine whether they had absolute pitch or not. The preliminary test consisted of 20 notes played on a piano. The subjects were required to identify the notes without comparing them with other tones.

$A P$ group. Five undergraduates were selected as subjects. Subject T.T. got full marks, and Subjects Y.I. and M.Y. got 19 out of 20. Subject N.Y. made three errors, wrongly identifying the notes as being one octave lower than they actually were. Subject Y.K. made three errors, identifying two of the mistaken notes as being one octave higher than they actually were.

Non-AP group. These subjects were also 5 undergraduate students. They received scores of 0 on the preliminary test. They had no known history of either speech or hearing disorders, and had little or no prior formal musical instruction.

Stimuli. Sine-wave tones were employed because stimulus tones produced by musical instruments contain cues other than pitch. Most of the subjects with absolute pitch who participated in this experiment played the piano. Bachem (1937) distinguished genuine absolute pitch from pseudo-absolute pitch. He described possessors of pseudo-absolute pitch as having the ability to identify tones only if they were accompanied by cues other than tone chroma, such as relative pitch and timbre. In the present study, it was feared that subjects might judge the similarity between the three stimuli on the basis of timbre, loudness, and so on, if piano tones were used.

Sine-wave tones were prepared on a VAX-8530 computer (DEC). They were normalized by using the overall maximum amplitude. All the stimuli were read out at a sampling rate of $10 \mathrm{kHz}$ with an accuracy of 12 bits, converted into analog waveform, and recorded on a magnetic tape through a low-pass filter with a cutoff frequency of $4.5 \mathrm{kHz}$ for off-line testing. All the stimuli had a steady duration of $200 \mathrm{msec}$, with rise and decay times of $20 \mathrm{msec}$.

One stimulus set consisted of three tones (A, X, and B). The experiments were conducted under two stimulus conditions. One was called the octave condition, in which the frequency separation between the two standard tones (A and B) was one octave, and the other was called the whole-tone condition, in which the frequency separation between A and B was a whole tone (a major second interval). In both conditions, ascending and descending stimulus orders were made.

Octave condition. In the ascending order, the frequencies of the first and third tones were held constant at $880 \mathrm{~Hz}$ (A5) and $1760 \mathrm{~Hz}$ (A6), respectively. The frequencies of the second tone were selected to divide the octave into eight musical intervals (just intonation); they were $990 \mathrm{~Hz}$ (B5), $1056 \mathrm{~Hz}$ (C6), $1188 \mathrm{~Hz}$ (D6), $1254 \mathrm{~Hz}$ (D\#6), $1320 \mathrm{~Hz}$ (E6), $1408 \mathrm{~Hz}$ (F6), and $1584 \mathrm{~Hz}$ (G6). Thus, seven types of stimulus triads were formed. Although equal temperament might have been preferable to just intonation in general (since most subjects acquired absolute pitch through piano training), the present study employed just intonation because of the following reasons: (1) some of the subjects who participated in the present experiments played the violin or belonged to a chorus group, (2) no transposition was required in the present experiments, (3) the subjects were expected to perceive the three tones as a stream of tones, (4) the subjects were required to make similarity judgments instead of identification, and (5) the stimuli were not piano tones but pure tones. For these reasons, it was thought that just intonation might be better than equal temperament for presenting the subjects with a more natural and comfortable stream of tones. The differences in frequency between equal temperament and just intonation are less than the differential limen for identification resolution by absolute pitch possessors (Burns, Campbell, \& Hoberg, 1989). After the experiments, no subjects reported that the tones seemed out of tune.

In the descending order, the first and third tones were fixed at $1760 \mathrm{~Hz}(\mathrm{~A} 6)$ and $880 \mathrm{~Hz}(\mathrm{~A} 5)$, respectively. The frequencies of the second tone were the same as those in the ascending order.

Whole-tone condition. In the ascending order, the frequencies of the first and third tones were held constant at $880 \mathrm{~Hz}$ (A5) and $990 \mathrm{~Hz}$ (B5), respectively. Seven stimuli, whose frequency range was from 890 to $980 \mathrm{~Hz}$, were selected to divide the frequency range into six equal intervals. The step size between adjacent stimuli was $15 \mathrm{~Hz}$. Over this range, the difference between equal and ratio intervals and the difference between the step intervals are so small that subjects ignored the differences.

Under these conditions, subjects with absolute pitch were considered to have two strategies. One strategy was to judge a target tone as lower or higher relative to the note (A\#5), which was halfway between the two standard tones (A5 and B5). In this case, the two standard tones and the halfway tone could be heard as anchors. With this strategy, no auditory tau effect could be observed in the whole-tone condition. The other strategy that subjects might employ was to use their auditory memory on pitch and, hence, suffer from a context effect caused by the temporal positions of previous or trailing tones, just as do subjects with nonabsolute pitch (Shigeno, 1986). With this strategy, the auditory tau effect could be observed.

In the descending order, the first and third tones were fixed at 990 and $880 \mathrm{~Hz}$, respectively. The frequencies of the second tone were the same as those in the ascending order.

Procedure. The experiment was conducted with the use of the AXB method. As is shown in Figure 1, three tones-A, X, and $B$-were presented successively. The subjects were required to judge whether the pitch of the second (target) tone $(X)$ was more similar to that of A or of B. It was further explained to the subjects that in this case "similar" meant "closer in pitch." Two successive short time intervals, represented as $\mathrm{tl}$ (between $\mathrm{A}$ and $\mathrm{X}$ ) and $\mathrm{t} 2$ (between $\mathrm{X}$ and $\mathrm{B}$ ), were delimited by the three tones and were varied by changing the temporal position of the second tone. Three temporal conditions, represented by the time ratio $t 1 /(t 1+t 2)$, were $.05, .50$, and .95 . Then 21 stimulus triads were made ( 7 target stimuli $\times 3$ temporal conditions). The total duration $(\mathrm{t} 1+\mathrm{t} 2)$ remained constant at $1.0 \mathrm{sec}$.

The same triad was judged by the subjects five times in one series. The subjects were given the stimulus series twice. To avoid the serial position effect, the initial three and the last two trials of each series were excluded from the data. Ten judgments for each of the seven targets were thus obtained from each subject for each of the three temporal conditions.

The recorded stimuli were played back at a comfortable listening level on a tape recorder (Sony, TC-365) through headphones (Bever Dynamic, DT48). Because the auditory tau effect has been 


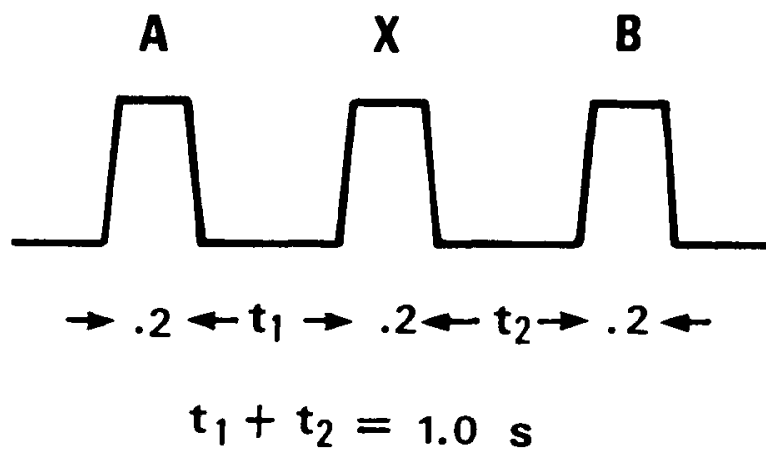

Figure 1. Presentation of stimuli in an AXB method.

found to occur both binaurally and monaurally (Cohen, Christensen, \& Ono, 1974), the stimuli were given to the subjects binaurally. Successive trials were separated by $3 \mathrm{sec}$, and there was a 5 -sec pause after every 10 trials.

\section{Results and Discussion}

The probability that the target tone would be judged as similar to either of the two other tones as a function of the tone frequency can generally be approximated by a cumulative normal distribution. Therefore the percentage of responses indicating that the target was similar to the third tone for the three temporal conditions for each subject was approximated by a cumulative normal distribution whose mean and standard deviation were obtained by the least squares method with Müller-Urban weight for each subject. The mean indicates the subjective middle frequency between the first and third tones. The standard deviation serves as an index for the accuracy of the judgments.

Tables 1 and 2 show the means and standard deviations for each of the absolute pitch possessors and of the nonabsolute pitch possessors in the octave and the whole-tone conditions, separately. Figures 2 and 3 show the pooled results (means and standard deviations) obtained from the 5 subjects in the AP group and the 5 subjects in the nonAP group. The results were plotted as a function of the time ratio condition of the second tone. The white circles represent the results of absolute pitch listeners, and the black circles represent the results of nonabsolute pitch listeners.

Octave condition (Table 1 and Figure 2). In the ascending order (the left graph of Figure 2), the subjective middle frequency of nonabsolute pitch listeners decreases as the second tone comes temporally closer to the third tone. This result can be understood to reflect the fact that when the second tone is put closer to the first (the lowest) tone, the subjects perceive the second tone as being lower than its actual frequency, and, thus, the subjective middle frequency is raised. When the second tone comes temporally closer to the third (the highest) tone, the subjects perceive the second tone as being higher than its actual frequency, and the subjective middle frequency is thus lowered. Consequently, the auditory tau effect (in this case, a linear downward trend) can be obtained.
The results were confirmed from the statistical analyses. A repeated measures one-way analysis of variance (ANOVA) with the factor of temporal position (3) was conducted. A significant effect for the series $[F(2,8)=$ $4.65, p=.0456]$ resulted.

In the descending order (the right graph of Figure 2), the auditory tau effect (in this case, a linear upward trend) is again obviously obtained in the perception of nonabsolute pitch possessors. The results seem to be asymmetrical between the ascending and the descending orders; the auditory tau effect seems to be more clearly shown in the ascending order than in the descending order, although the auditory tau effect was significantly observed in both of the stimulus orders. A repeated measures one-way ANOVA with the factor of temporal position (3) yielded a significant effect $[F(2,8)=13.41, p=.0028]$.

On the other hand, the subjective middle frequency of absolute pitch listeners remained constant both in the ascending and the descending orders. The results show that the pitch perception of the second tone was almost constant, regardless of the temporal separation in both the ascending and the descending orders; that is, no auditory tau effect occurred in the pitch judgment of the subjects with absolute pitch.

Two separate repeated measures two-way ANOVAs with the factors temporal position (3) and subject group

Table 1

The Subjective Middle Frequency and Standard Deviation (in Hertz) for Each Subject in the Octave Condition

\begin{tabular}{|c|c|c|c|c|c|c|c|}
\hline \multicolumn{2}{|c|}{ Subject } & \multicolumn{6}{|c|}{ Value of $t 1 /\left(t 1+t^{2}\right)$} \\
\hline \multirow{2}{*}{$\begin{array}{l}\text { Absolute } \\
\text { Pitch }\end{array}$} & \multirow{2}{*}{$\begin{array}{c}\text { Nonabsolute } \\
\text { Pitch }\end{array}$} & \multicolumn{2}{|c|}{.05} & \multicolumn{2}{|c|}{.50} & \multicolumn{2}{|c|}{.95} \\
\hline & & $M$ & $S D$ & $M$ & $S D$ & $M$ & $S D$ \\
\hline \multicolumn{8}{|c|}{ Ascending Order } \\
\hline $\begin{array}{l}\text { Y.I. } \\
\text { Y.K. } \\
\text { T.T. } \\
\text { N.Y. } \\
\text { M.Y. }\end{array}$ & & $\begin{array}{l}1287 \\
1287 \\
1272 \\
1236 \\
1221\end{array}$ & $\begin{array}{l}20 \\
26 \\
40 \\
40 \\
20\end{array}$ & $\begin{array}{l}1287 \\
1276 \\
1269 \\
1242 \\
1221\end{array}$ & $\begin{array}{l}20 \\
27 \\
60 \\
40 \\
20\end{array}$ & $\begin{array}{l}1287 \\
1270 \\
1272 \\
1236 \\
1221\end{array}$ & $\begin{array}{l}20 \\
30 \\
40 \\
40 \\
20\end{array}$ \\
\hline Mean & & 1261 & 30 & 1259 & 33 & 1257 & 30 \\
\hline & $\begin{array}{l}\text { K.I. } \\
\text { A.S. } \\
\text { N.S. } \\
\text { N.T. } \\
\text { I.H. }\end{array}$ & $\begin{array}{l}1340 \\
1639 \\
1272 \\
1260 \\
1207\end{array}$ & $\begin{array}{l}144 \\
305 \\
308 \\
291 \\
206\end{array}$ & $\begin{array}{l}1266 \\
1253 \\
1269 \\
1268 \\
1280\end{array}$ & $\begin{array}{l}100 \\
137 \\
217 \\
277 \\
176\end{array}$ & $\begin{array}{l}1230 \\
1171 \\
1155 \\
1113 \\
1088\end{array}$ & $\begin{array}{r}74 \\
158 \\
183 \\
186 \\
118\end{array}$ \\
\hline & Mean & 1344 & 251 & 1267 & 181 & 1151 & 144 \\
\hline \multicolumn{8}{|c|}{ Descending Order } \\
\hline $\begin{array}{l}\text { Y.I. } \\
\text { Y.K. } \\
\text { T.T. } \\
\text { N.Y. } \\
\text { M.Y. }\end{array}$ & & $\begin{array}{l}1276 \\
1272 \\
1233 \\
1221 \\
1221\end{array}$ & $\begin{array}{l}40 \\
40 \\
61 \\
20 \\
20\end{array}$ & $\begin{array}{l}1271 \\
1272 \\
1221 \\
1221 \\
1221\end{array}$ & $\begin{array}{l}49 \\
40 \\
20 \\
20 \\
20\end{array}$ & $\begin{array}{l}1273 \\
1272 \\
1232 \\
1221 \\
1221\end{array}$ & $\begin{array}{l}49 \\
40 \\
27 \\
20 \\
20\end{array}$ \\
\hline Mean & & 1245 & 36 & 1241 & 30 & 1244 & 31 \\
\hline & $\begin{array}{l}\text { K.I. } \\
\text { A.S. } \\
\text { N.S. } \\
\text { N.T. } \\
\text { I.H. }\end{array}$ & $\begin{array}{l}1267 \\
1155 \\
1255 \\
1175 \\
1086\end{array}$ & $\begin{array}{l}158 \\
208 \\
193 \\
165 \\
271\end{array}$ & $\begin{array}{l}1272 \\
1198 \\
1245 \\
1305 \\
1180\end{array}$ & $\begin{array}{r}81 \\
157 \\
180 \\
337 \\
128\end{array}$ & $\begin{array}{l}1331 \\
1251 \\
1332 \\
1415 \\
1281\end{array}$ & $\begin{array}{l}192 \\
190 \\
218 \\
263 \\
135\end{array}$ \\
\hline & Mean & 1188 & 199 & 1240 & 177 & 1322 & 200 \\
\hline
\end{tabular}


Table 2

The Subjective Middle Frequency and Standard Deviation (in Hertz) for Each Subject in the Whole-Tone Condition

\begin{tabular}{|c|c|c|c|c|c|c|c|}
\hline \multicolumn{2}{|c|}{ Subject } & \multicolumn{6}{|c|}{ Value of $\mathrm{tl} /(\mathrm{t} 1+\mathrm{t} 2)$} \\
\hline Absolute & Nonabsolute & \multicolumn{2}{|c|}{.05} & \multicolumn{2}{|c|}{.50} & \multicolumn{2}{|c|}{.95} \\
\hline Pitch & Pitch & $M$ & $S D$ & $M$ & $S D$ & $M$ & $S D$ \\
\hline \multicolumn{8}{|c|}{ Ascending Order } \\
\hline $\begin{array}{l}\text { Y.I. } \\
\text { Y.K. } \\
\text { T.T. } \\
\text { N.Y. } \\
\text { M.Y. }\end{array}$ & & $\begin{array}{l}962 \\
945 \\
935 \\
948 \\
945\end{array}$ & $\begin{array}{r}9 \\
21 \\
18 \\
34 \\
47\end{array}$ & $\begin{array}{l}961 \\
937 \\
953 \\
930 \\
935\end{array}$ & $\begin{array}{c}8 \\
33 \\
31 \\
38 \\
38\end{array}$ & $\begin{array}{l}953 \\
922 \\
953 \\
908 \\
918\end{array}$ & $\begin{array}{l}21 \\
22 \\
31 \\
13 \\
18\end{array}$ \\
\hline Mean & & 947 & 26 & 943 & 30 & 931 & 21 \\
\hline & $\begin{array}{l}\text { K.I. } \\
\text { A.S. } \\
\text { N.S. } \\
\text { N.T. } \\
\text { I.H. }\end{array}$ & $\begin{array}{l}932 \\
939 \\
967 \\
930 \\
928\end{array}$ & $\begin{array}{l}27 \\
24 \\
55 \\
31 \\
31\end{array}$ & $\begin{array}{l}936 \\
936 \\
940 \\
937 \\
947\end{array}$ & $\begin{array}{l}24 \\
32 \\
29 \\
37 \\
16\end{array}$ & $\begin{array}{l}928 \\
939 \\
916 \\
904 \\
923\end{array}$ & $\begin{array}{l}28 \\
19 \\
58 \\
16 \\
14\end{array}$ \\
\hline & Mean & 939 & 34 & 939 & 28 & 922 & 27 \\
\hline \multicolumn{8}{|c|}{ Descending Order } \\
\hline $\begin{array}{l}\text { Y.I. } \\
\text { Y.K. } \\
\text { T.T. } \\
\text { N.Y. } \\
\text { M.Y. }\end{array}$ & & $\begin{array}{l}910 \\
916 \\
923 \\
916 \\
926\end{array}$ & $\begin{array}{r}14 \\
9 \\
42 \\
8 \\
16\end{array}$ & $\begin{array}{l}910 \\
927 \\
929 \\
916 \\
935\end{array}$ & $\begin{array}{l}16 \\
39 \\
49 \\
43 \\
15\end{array}$ & $\begin{array}{l}922 \\
950 \\
935 \\
951 \\
939\end{array}$ & $\begin{array}{l}20 \\
26 \\
40 \\
30 \\
28\end{array}$ \\
\hline Mean & & 918 & 18 & 923 & 32 & 939 & 29 \\
\hline & $\begin{array}{l}\text { K.I. } \\
\text { A.S. } \\
\text { N.S. } \\
\text { N.T. } \\
\text { I.H. }\end{array}$ & $\begin{array}{l}928 \\
938 \\
937 \\
924 \\
940\end{array}$ & $\begin{array}{r}20 \\
29 \\
133 \\
27 \\
59\end{array}$ & $\begin{array}{l}933 \\
929 \\
953 \\
939 \\
906\end{array}$ & $\begin{array}{l}26 \\
29 \\
81 \\
39 \\
56\end{array}$ & $\begin{array}{l}954 \\
934 \\
950 \\
959 \\
958\end{array}$ & $\begin{array}{l}29 \\
25 \\
77 \\
16 \\
36\end{array}$ \\
\hline & Mean & 933 & 54 & 932 & 46 & 951 & 37 \\
\hline
\end{tabular}

(2) yielded significant interactions for the ascending order $[F(2,16)=4.48, p=.0284]$ and the descending order $[F(2,16)=13.42, p=.0004]$. There was no specific trend in standard deviations between the stimulus orders, although the standard deviations of nonabsolute pitch possessors were much greater than those of absolute pitch possessors.

Whole-tone condition (Table 2 and Figure 3). In the ascending order (the left graph of Figure 3), the subjective middle frequency of nonabsolute pitch listeners decreases as the second tone comes temporally closer to the third tone. This result can be accounted for in the following way: when the second tone is temporally closer to the first (the lowest) tone, the subjects perceive the second tone as being lower than its actual frequency, and, thus, the subjective middle frequency is raised. When the second tone is temporally closer to the third (the highest) tone, the subjects perceive the second tone as being higher than its actual frequency, and the subjective middle frequency is thus lowered. Accordingly, the auditory tau effect is suggested. Analogously, in the descending stimulus order (the right half of Figure 3), the auditory tau effect is suggested from the responses of nonabsolute pitch listeners.

Two separate repeated measures one-way ANOVAs with the factor of temporal position (3), however, yielded no significant effect for the ascending order $[F(2,8)=$
$3.08, p=.1017]$ or for the descending order $[F(2,8)=$ $3.08, p=.1019]$. The results here do not show the auditory tau effect as clearly as in the octave condition. One possible cause is the stimulus range over the standard tones. As Cohen et al. (1954) pointed out, the auditory tau effect is larger when the difference between the outer tones is greater. There was no specific trend in standard deviations between the stimulus series, although the standard deviations of nonabsolute pitch possessors were a little greater than those of absolute pitch possessors.

In both the ascending and the descending orders, the auditory tau effects are also suggested by the responses of absolute pitch listeners. Two separate repeated measures one-way ANOVAs with the factor of temporal position (3) did not yield a significant effect for the ascend-

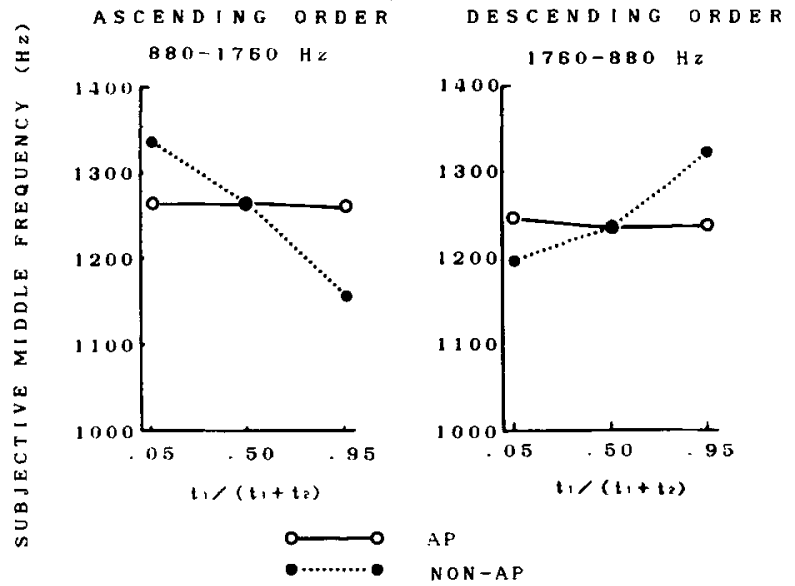

Figure 2. The subjective middle frequency (in Hertz) of the pooled data for all the subjects, as a function of the time ratio condition of the second (target) tone for the octave condition when the stimulus continuum consisted of musical notes.

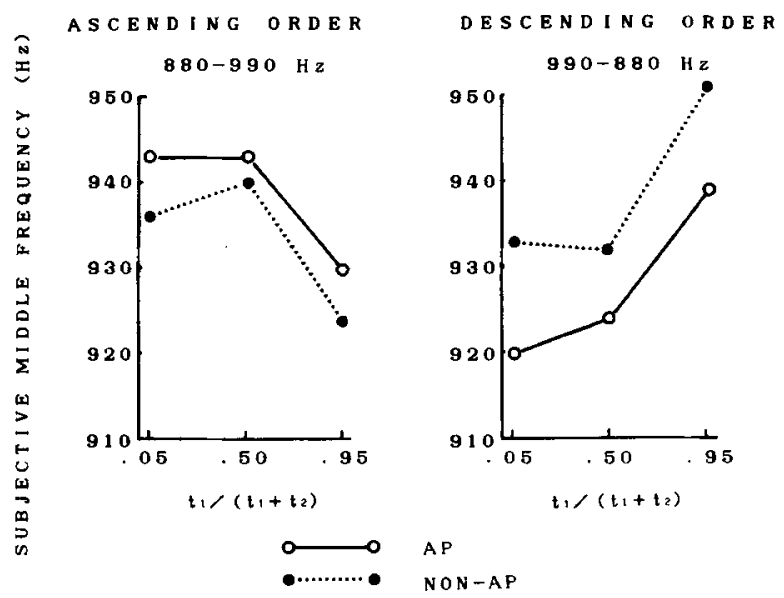

Figure 3. The subjective middle frequency (in Hertz) of the pooled data for all the subjects, as a function of the time ratio condition of the second (target) tone for the whole-tone condition, where the stimulus continuum consisted of tones that did not correspond to musical notes. 
ing order $[F(2,8)=2.89, p=.1139]$, but yielded a significant effect for the descending order $[F(2,8)=10.74$, $p<.0054$ ]. Two separate repeated measures two-way ANOVAs with the factors temporal position (3) and subject group (2) yielded no significant interaction for the ascending order $[F(2,16)=0.11, p=.8940]$ or the descending order $[F(2,16)=0.23, p=.7982]$. Since there were no significant interactions between the subjects with absolute pitch and those with nonabsolute pitch, the data from the two groups were combined. Two separate repeated measures one-way ANOVAs with the factor of temporal position (3) yielded significant effects of ascending order $[F(2,18)=6.52, p=.0074]$ and of descending order $[F(2,18)=10.48, p=.0010]$.

Figure 3 also shows that in the judgments of both absolute pitch and nonabsolute pitch listeners the results are asymmetrical between the ascending and descending orders. The results further indicate that the judgment of absolute pitch listeners was influenced by the preceding tones more than by the trailing tones. More precisely, even when the second tone was temporally mediate, they apparently heard it as sounding flat in the ascending order and sharp in the descending order. Further investigation may be necessary to explain this finding precisely.

We can interpret the present results as follows. When the target tones correspond to musical notes, the subjects with absolute pitch perceive the pitch of the tones by using absolute pitch. They first identify the tones as specific musical notes, and then judge the similarities between the three tones on the basis of the musical notes. Because their judgments are absolute, they do not suffer from a context effect. On the other hand, when the target tones do not correspond to musical notes, the subjects with absolute pitch perceive the pitch of the tones on the basis of their auditory memory of pitch of context tones, and therefore they suffer from a context effect caused by temporal factors, just as do subjects with nonabsolute pitch. The asymmetry between the ascending and the descending orders and the nonlinear results in the whole-tone condition might be based on the differences in the magnitudes of forward and backward context effects (Shigeno, 1986).

The results can also be explained by the hypothesis proposed by Siegel (1974) that possessors of absolute pitch have distinct modes for processing tone frequency-a sensory trace mode that maintains their sensory event for a very brief time in memory, and a verbal mode, in which tones are labeled with musical note names. When the target tones correspond to musical notes, the verbal mode is mainly used. When the target tones do not correspond to musical notes, the sensory trace mode is mainly used. Subjects with nonabsolute pitch make use of the latter mode.

In the whole-tone condition, subjects with absolute pitch did not use the strategy of referring to the note (A\#5) that was halfway between the two context tones (A and B) as an anchor to judge a target tone to be lower or higher. Instead of this strategy, they might use their auditory memory of pitch and, hence, suffer from a context effect caused by the temporal positions of previous or trailing tones, just as did subjects with nonabsolute pitch. The whole temporal duration of three successive tones was $1 \mathrm{sec}$. It was rather difficult for the subjects to imagine the pitch of the note (A\#5) and keep it in memory as an anchor. This speculation was also confirmed by the introspection obtained from 1 of the subjects. Subject T.T. reported, "I could hardly imagine the note $A \#$, because there were so many targets in the test and I couldn't identify which one was the real A\#. I was too busy identifying each of the tones. I listened to the three tones as a series of tones; then I judged the similarity." Accordingly, the whole-tone might cover two (not three) categories in Experiment 1. The categorical relationship between the stimuli in the whole-tone condition might correspond to that in the between-category condition (e.g., /i/-/e/) in the speech experiment (Shigeno, 1986).

In the similarity judgment of speech, Shigeno (1986) found that in the same situation as in pitch judgment of the whole-tone condition, the subjects tend to judge the second vowels contrastively in relation to the temporally closer sound (the reverse pattern of the auditory tau effect). On the basis of these experimental results, Shigeno hypothesized that the hierarchical auditory information processing is influenced by temporal factors (temporal separation between stimuli) at two different perceptual levels: the auditory (precategorical) and the phonetic (categorical). Shigeno's results were quite different from those obtained in the whole-tone condition in Experiment 1. In the present experiment, when possessors of absolute pitch made similarity judgments of pitch, they showed assimilation, not contrast. These results demonstrated that although the pitch judgments of absolute pitch possessors are retained in a categorical mode as are the vowel judgments, the context produces different effects-assimilation (the auditory tau effect) in the case of absolute pitch judgment and contrast (the reverse pattern of the auditory tau effect) in the case of vowel judgment. Shigeno (1987) obtained the auditory tau effect in the perception of voiced stop consonants, using the stimulus continua that consist of "between-phonetic-category" stimuli. The mechanisms of these effects have not been demonstrated conclusively. Shigeno (1987) suggested that the mechanism of the auditory tau effect may be described only in terms of a model that can explain the mechanisms of both assimilation and contrast.

\section{EXPERIMENT 2}

In the octave condition of Experiment 1, where the frequencies of the second tone divided the octave interval into eight musical intervals, the subjects with absolute pitch showed neither the auditory tau effect nor the reverse pattern of the auditory tau effect. Their perception was hardly influenced by the context. But in the wholetone condition, they showed the auditory tau effect. It can be suggested that they might change their strategy from using absolute pitch in the octave condition to using the auditory memory of pitch in the whole-tone condition, just 
as nonabsolute pitch possessors do. The results in Experiment 1, however, may be due to the different ranges of pitch, such as octave and whole-tone intervals. Experiment 2 was an attempt to examine the dependence of pitch judgment on temporal separation, with the second tone chosen so as not to correspond to any musical note within an octave interval.

\section{Method}

Subjects. The subjects were 5 undergraduate students with absolute pitch, who had participated in Experiment 1.

Stimuli. Sine-wave tones were used. They were prepared with the same method as in Experiment 1. Only the octave condition was utilized. In the ascending order, the first and third tones were fixed at 880 and $1760 \mathrm{~Hz}$, respectively. The second (target) tone varied from 1020 to $1620 \mathrm{~Hz}$ at the equal interval of $100 \mathrm{~Hz}$. In the descending order, the first and third tones were 1760 and $880 \mathrm{~Hz}$, respectively. The second tones were the same as those in the ascending order.

Procedure. The procedure was practically the same as that of Experiment 1.

\section{Results and Discussion}

Table 3 shows the results obtained from each of the subjects. Figure 4 shows the pooled results obtained from the 5 subjects. The results were plotted as a function of the time ratio condition of the second tone. In both the ascending and the descending orders, the subjective middle frequency was around $1259 \mathrm{~Hz}$-a little higher than the geometric mean $(1245 \mathrm{~Hz})$. The results indicate that under the octave interval condition-even when the target tones did not correspond to musical notes-the pitch judgment of possessors of absolute pitch was not influenced at all by the context of temporal intervals. The standard deviations were almost constant, although the standard deviations of the descending order were greater than those of the ascending order in some of the subjects.

Table 3

The Subjective Middle Frequency and Standard Deviation (in Hertz) for Each Subject With Absolute Pitch in the Octave Condition

\begin{tabular}{|c|c|c|c|c|c|c|}
\hline \multirow[b]{3}{*}{ Subject } & \multicolumn{6}{|c|}{ Value of $t 1 /(t 1+t 2)$} \\
\hline & \multicolumn{2}{|c|}{.05} & \multicolumn{2}{|c|}{.50} & \multicolumn{2}{|c|}{.95} \\
\hline & $M$ & $S D$ & $M$ & $S D$ & $M$ & $S D$ \\
\hline \multicolumn{7}{|c|}{ Ascending Order } \\
\hline $\begin{array}{l}\text { Y.I. } \\
\text { Y.K. } \\
\text { T.T. } \\
\text { N.Y. } \\
\text { M.Y. }\end{array}$ & $\begin{array}{l}1270 \\
1276 \\
1270 \\
1244 \\
1270\end{array}$ & $\begin{array}{l}30 \\
34 \\
30 \\
46 \\
30\end{array}$ & $\begin{array}{l}1270 \\
1270 \\
1263 \\
1254 \\
1264\end{array}$ & $\begin{array}{l}30 \\
30 \\
34 \\
40 \\
34\end{array}$ & $\begin{array}{l}1270 \\
1263 \\
1270 \\
1233 \\
1244\end{array}$ & $\begin{array}{l}30 \\
34 \\
30 \\
53 \\
46\end{array}$ \\
\hline Mean & 1266 & 34 & 1264 & 34 & 1256 & 39 \\
\hline \multicolumn{7}{|c|}{ Descending Order } \\
\hline $\begin{array}{l}\text { Y.I. } \\
\text { Y.K. } \\
\text { T.T. } \\
\text { N.Y. } \\
\text { M.Y. }\end{array}$ & $\begin{array}{l}1270 \\
1254 \\
1247 \\
1264 \\
1229\end{array}$ & $\begin{array}{l}30 \\
40 \\
78 \\
34 \\
61\end{array}$ & $\begin{array}{l}1270 \\
1270 \\
1264 \\
1270 \\
1202\end{array}$ & $\begin{array}{l}30 \\
30 \\
34 \\
30 \\
61\end{array}$ & $\begin{array}{l}1270 \\
1270 \\
1269 \\
1270 \\
1220\end{array}$ & $\begin{array}{r}30 \\
30 \\
192 \\
30 \\
61\end{array}$ \\
\hline Mean & 1253 & 49 & 1255 & 37 & 1260 & 69 \\
\hline
\end{tabular}

Note-The second tone was chosen so that it would not correspond to any musical note.
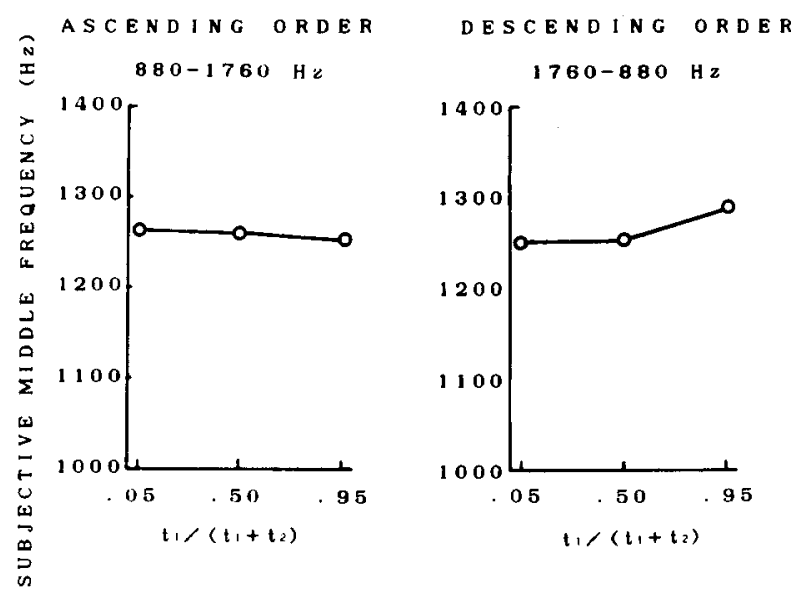

Figure 4. The subjective middle frequency (in Hertz) of the pooled data for all the subjects, as a function of the time ratio condition of the second (target) tone for the octave condition when the stimulus continuum consisted of the tones that did not correspond to musical notes.

It is possible that possessors of absolute pitch perceive the second tone as corresponding to the nearest musical notes. The results in Experiment 2 can be explained by the previous findings. Ward and Burns (1982) showed that good absolute pitch possessors can easily identify out-oftune notes to the nearest quarter tone. Siegel and Siegel (1977b) found that in the magnitude estimation task, musicians had a strong tendency to rate out-of-tune stimuli as being in tune. Miyazaki (1988) showed that genuine absolute pitch possessors can easily and immediately identify the tones with absolute pitch under conditions designed to minimize the possibility that subjects could use strategies other than absolute pitch in performing the task. This interpretation is further supported by the introspections obtained from the subjects. Most of them reported that the second tones had consisted of musical notes with sharps and flats.

\section{EXPERIMENT 3}

The kappa effect (an effect of sensory differences on the perception of temporal intervals) has been regarded as the converse of the tau effect (an effect of temporal intervals on the perception of spatial separation or sensory difference). Shigeno (1986) suggested that pitch and vowel judgment is influenced by temporal factors (temporal separation between two stimuli) at the precategorical and categorical levels, and that temporal perception is influenced by spatial factors (spatial separation on the stimulus continuum between two stimuli) at the precategorical level, but not at the categorical level. The experimental results obtained so far support the idea that the time-space (or time-phonetic category) interaction is asymmetrical; that is, the auditory tau effect is a kind of context effect and is not always obtained in the cases 
where the kappa effect is clearly found. The purpose of Experiment 3 was to explore further aspects of the time-pitch relation in auditory judgment. The question is whether or not the kappa effect occurs in the temporal perception of possessors of absolute pitch.

\section{Method}

Subjects. The subjects were 5 undergraduates with absolute pitch, who had participated in Experiments 1 and 2.

Stimuli. Sine-wave tones were used. The stimuli were prepared with the same method as in Experiments 1 and 2. The octave and the whole-tone conditions, which were used in Experiment 1, were employed.

Octave condition. In the ascending order, the first and third tones were fixed at 880 and $1760 \mathrm{~Hz}$, respectively. In Experiment 3, the second (target) tone was 990,1254 , or $1584 \mathrm{~Hz}$. In the descending order, the first and third tones were fixed at 1760 and $880 \mathrm{~Hz}$, respectively. The second tone was the same as that in the ascending order.

Whole-tone condition. In the ascending order, the first and third tones were fixed at 880 , and $990 \mathrm{~Hz}$, respectively. In Experiment 3, the second tone was 890,935 , or $980 \mathrm{~Hz}$. In the descending order, the first and third tones were fixed at 990 and $880 \mathrm{~Hz}$, respectively. The second tone was the same as that in the ascending order.

Procedure. The AXB method was employed. In this experiment, the subjects were asked to judge whether the second (target) tone was closer to A or to B in time, not in pitch. The interstimulus intervals between the first and second tones and between the second and third tones were denoted as $t 1$ and 2 , respectively. The seven temporal conditions, represented by the time ratio $t 1 /(t 1+t 2)$, were $.41, .44, .47, .50, .53, .56$, and .59 . As a result, 21 stimulus triads were made in each stimulus series ( 7 temporal conditions $\times$ 3 target stimuli). The total duration $(\mathrm{t} 1+\mathrm{t} 2)$ was constant at $1.0 \mathrm{sec}$.

The same triad was judged by the subjects five times in one series. The subjects in each condition were given the same stimulus series twice. The initial three and the last two trials of each stimulus series were excluded from the data. Ten judgments for each second tone were thus obtained from each subject for each of the three frequency conditions of the second tone.

The recorded stimuli were played back on a tape recorder (Sony TC-365) and presented to the subjects in a quiet room through headphones (Bever Dynamic, DT48) at a comfortable listening level.

\section{Results and Discussion}

The means and standard deviations were obtained by the least squares method with Müller-Urban weight for each subject. The mean corresponds to the subjective middle temporal point between the first and third tones along the time axis.

Table 4 shows the means and standard deviations for each of the absolute pitch possessors and of the nonabsolute pitch possessors, separately. Figure 5 shows the pooled results obtained from the 5 subjects. The results were plotted as a function of the frequency of the second (target) tone.

Octave condition (the upper panels of Figure 5). In the ascending order (the left graph), an examination of the curve shows that when the frequency of the second tone is lower than the geometric mean of the first and third tones $(1245 \mathrm{~Hz})$, the interval $\mathrm{tl}$ is perceived to be shorter than it actually is; hence the subjective middle temporal point is greater than the objective one $(500 \mathrm{msec})$. When the frequency of the second tone approaches the geomet-
Table 4

The Subjective Middle Temporal Point and Standard Deviation (in Milliseconds) for Each Subject With Absolute Pitch

\begin{tabular}{|c|c|c|c|c|c|c|c|c|c|c|c|c|}
\hline \multirow[b]{4}{*}{ Subject } & \multicolumn{12}{|c|}{ Second-Tone Frequency } \\
\hline & \multicolumn{6}{|c|}{ Octave Condition } & \multicolumn{6}{|c|}{ Whole-Tone Condition } \\
\hline & \multicolumn{2}{|c|}{990} & \multicolumn{2}{|c|}{1254} & \multicolumn{2}{|c|}{1584} & \multicolumn{2}{|c|}{890} & \multicolumn{2}{|c|}{935} & \multicolumn{2}{|c|}{980} \\
\hline & $M$ & $S D$ & $M$ & $S D$ & $M$ & $S D$ & $M$ & $S D$ & $M$ & $S D$ & $M$ & $S D$ \\
\hline \multicolumn{13}{|c|}{ Ascending Order } \\
\hline Y.I. & 560 & 61 & 508 & 62 & 456 & 61 & 519 & 124 & 537 & 49 & 481 & 94 \\
\hline Y.K. & 539 & 40 & 526 & 27 & 446 & 51 & 495 & 53 & 502 & 37 & 457 & 32 \\
\hline T.T. & 550 & 58 & 540 & 61 & 469 & 55 & 515 & 56 & 505 & 63 & 496 & 54 \\
\hline N.Y. & 561 & 77 & 500 & 80 & 441 & 64 & 544 & 33 & 533 & 48 & 525 & 40 \\
\hline M.Y. & 515 & 42 & 496 & 46 & 474 & 59 & 500 & 46 & 523 & 84 & 479 & 17 \\
\hline Mean & 545 & 56 & 514 & 55 & 457 & 58 & 515 & 62 & 520 & 56 & 488 & 47 \\
\hline \multicolumn{13}{|c|}{ Descending Order } \\
\hline Y.I. & 479 & 59 & 513 & 64 & 514 & 68 & 460 & 74 & 517 & 73 & 548 & 54 \\
\hline Y.K. & 444 & 36 & 471 & 65 & 518 & 47 & 460 & 23 & 490 & 51 & 523 & 42 \\
\hline T.T. & 473 & 53 & 500 & 46 & 522 & 43 & 513 & 53 & 504 & 54 & 537 & 50 \\
\hline N.Y. & 441 & 64 & 511 & 96 & 551 & 69 & 521 & 63 & 511 & 37 & 545 & 42 \\
\hline M.Y. & 488 & 36 & 496 & 27 & 500 & 28 & 490 & 32 & 512 & 14 & 538 & 23 \\
\hline Mean & 465 & 50 & 498 & 60 & 521 & 51 & 489 & 49 & 507 & 46 & 538 & 42 \\
\hline
\end{tabular}

ric mean of the first and third, the perceived temporal interval $t 1$ approaches the actual temporal interval. When the frequency of the second tone is higher than the geometric mean, the temporal interval $t l$ is perceived to be longer than the actual temporal interval. Accordingly, the subjective middle temporal point is smaller than the objective one. The results show that the kappa effect (in this case, a linear downward trend) occurs in the temporal perception of the subjects with absolute pitch. In the descending order (the right graph), the kappa effect (in this case a linear upward trend) may also be observed, although the amount of the effect is a little smaller than that in the ascending order.

Two separate repeated measures one-way ANOVAs with the factor of the frequency of the second tone (3) yielded significant effects of the ascending order $[F(2,8)=30.54, p=.0002]$ and the descending order $[F(2,8)=10.08, p=.0065]$. There was no specific trend in standard deviations between the ascending and descending orders.

Whole-tone condition (the lower panels of Figure 5). In the ascending order (the left graph), an examination of the curve shows that when the frequency of the second tone is lower than the geometric mean between the first and third tones $(933 \mathrm{~Hz})$, the interval $\mathrm{tl}$ is perceived to be shorter than it actually is; hence, the subjective middle temporal point is greater than the objective one $(500 \mathrm{msec})$. When the frequency of the second stimulus approaches the geometric mean, the perceived temporal interval $t 1$ is still perceived to be shorter than the actual interval. When the frequency of the second stimulus is higher than the geometric mean, the temporal interval $t l$ approaches the actual temporal interval. The results show that the kappa effect (in this case, a linear downward trend) occurs in the temporal perception of the subjects with absolute pitch, although the tendency is not so strong. 

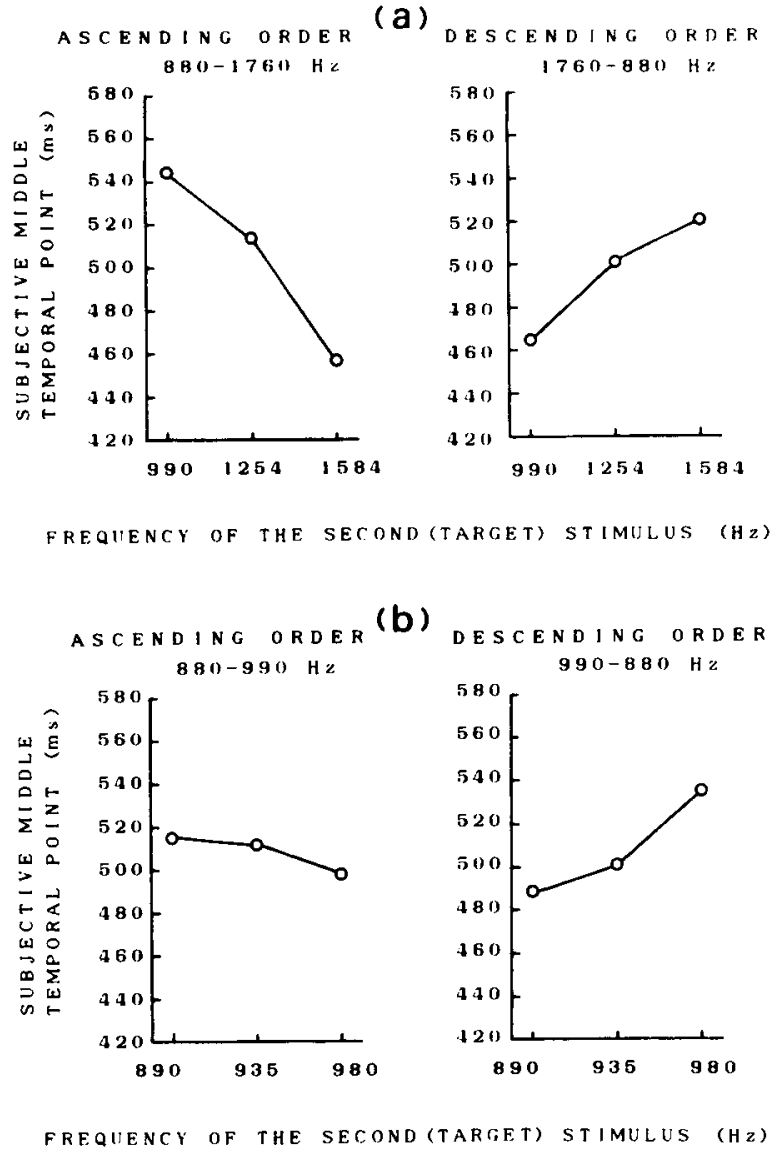

Figure 5. The subjective middle temporal point in milliseconds of the pooled data for all the subjects, as a function of the second stimulus. (a) The octave condition; (b) the whole-tone condition.

In the descending order (the right graph), the kappa effect (a linear upward trend) may be observed more clearly.

Two separate repeated measures one-way ANOVAs with the factor of the frequency of the second tone (3) yielded significant effects for the ascending order $[F(2,8)=10.71, p=.0055]$ and the descending order $[F(2,8)=12.09, p=.0038]$.

The standard deviations fell between 14 and $124 \mathrm{msec}$. The standard deviations of the ascending order were somewhat greater than those of the descending order. This might be the cause of the weaker tendency of the kappa effect in the ascending order as opposed to the descending order.

The kappa effect was greater in the octave condition than in the whole-tone condition in the ascending order and almost equal in the descending order. Therefore, the range of stimulus frequencies and the stimulus presentation orders are the factors that determine the degree of the kappa effect. Shigeno (1986) found asymmetry between stimulus series in the auditory tau effect and proposed that the auditory tau effect is a context effect that integrates forward and backward context effects. The asymmetry in temporal perception can also be explained if one sees that the kappa effect is one of the context effects caused by the integration of forward and backward context effects within one triad. In speech perception, Repp, Healy, and Crowder (1979) and Shigeno and Fujisaki (1980) found that backward contrast was stronger than forward contrast in phonetic judgments of isolated synthetic vowels. Shigeno and Fujisaki (1980) found that nonphonetic (pitch) judgments of isolated synthetic vowels exhibited smaller contrast effects and no (or the opposite) difference between forward and backward contrast. The magnitude of the forward and backward context effects may vary with the vowel categories used as context stimuli and may cause the asymmetry of the auditory tau effect. Similarly, the kappa effect may be explained in terms of the differences in the magnitudes of forward and backward context effects in temporal perception. However, the present results cannot give us any conclusive answer to the question of why the amount of the kappa effect is different according to the stimulus range and the stimulus order.

Although the auditory tau effect in the perception of absolute pitch possessors was observed under the wholetone condition but not under the octave condition, the kappa effect was observed under both the octave and the whole-tone conditions in the perception of absolute pitch possessors. Parks (1968) studied the kappa effect in vision and proposed that the kappa effect reflects not postperceptual processes, but a disturbance in the memorytrace of the temporal extent of the test interval. The results of Experiment 3 support these speculations. Shigeno (1986) indicated that the kappa effect occurred for both speech and nonspeech stimuli, and proposed that the kappa effect occurs at a common stage of auditory information processing before phonetic categorization. The results of the present study, in which the kappa effect was obtained in both the octave and the whole-tone condition, may provide further evidence that the kappa effect in the perception of absolute pitch possessors occurs before the categorization into musical note names.

\section{GENERAL DISCUSSION}

The present study presents four major results: (1) In the pitch judgment of possessors of absolute pitch, the auditory tau effect was observed to be significant when the stimulus continuum consisted of tones that did not correspond to musical notes in the whole-tone condition, but was not observed at all when the stimulus continuum consisted of tones that corresponded to or did not correspond to musical notes in the octave condition. In the latter case, the pitch judgment of the subjects with absolute pitch was constant, irrespectively of the temporal separation of the three tones. (2) In the pitch perception of the subjects with nonabsolute pitch, the auditory tau effect was observed to be significant both when the stimulus continuum consisted of musical notes in the octave interval and when it consisted of tones that did not correspond to musical notes in the whole-tone interval. (3) The magnitude of the 
auditory tau effect was not symmetrical between the ascending and the descending stimulus orders. (4) The kappa effect in the temporal perception of absolute pitch possessors was obtained for the ascending and the descending stimulus orders in both the octave and the wholetone intervals.

\section{Time-Space Interdependence}

The results of the present experiment suggest that the auditory tau effect occurs at the precategorical level, but not at the categorical level; the tau effect was not observed in the absolute pitch possessors who could categorize pitch into musical notes. This indicates that the hierarchical auditory information processing is influenced by temporal factors (temporal position of previous and trailing tones) at two different perceptual levels-the precategorical (auditory), and the categorical. The present study supports the idea proposed by Shigeno (1991) that the strategy for similarity judgment depends on whether the stimuli belong to the same category or to different categories. The subjects would make use of the information on timbre when categorical information was not available.

The results from the experiment on the kappa effect, on the other hand, show that temporal perception is influenced by spatial factors (pitch separation on the stimulus continuum between stimuli) at the level prior to categorical judgment - that is, at the acoustic information processing level. Although the kappa effect has been regarded as the converse phenomenon of the tau effect and the investigations on the tau and kappa effects suggest that there is a tradeoff between temporal and spatial perception, the present study suggests that the auditory tau and the kappa effects are not precisely converse phenomena. The auditory tau effect depends on the categorical relationship between the stimuli and the strategy employed by the subjects. The kappa effect, however, is not influenced by these factors. In this sense, the time-space interaction may be asymmetrical.

\section{Pitch and Temporal Judgments of Possessors of Absolute Pitch}

It has been suggested that categorical perception is unique to speech (Liberman, Cooper, Shankweiler, \& Studdert-Kennedy, 1967). Yet categorical perception of nonspeech has often been used to demonstrate that categorical perception is not unique to speech perception (see the introduction). Siegel and Siegel (1977b) demonstrated that musicians with good relative pitch can label tonal intervals accurately on an absolute basis. The naming distributions are symmetrical, with well-defined category boundaries and little overlap between adjacent categories, resembling what we usually find with speech perception. In one recent study, Burns et al. (1989) demonstrated that absolute pitch can be considered an example of categorical perception only to the extent that identification resolution for frequency is similar to that for several categor- ically perceived continua, since it does not meet the other criterion that usually defines categorical perception (i.e., possessors of absolute pitch clearly can discriminate many more stimuli than they can identify).

The present research indicates that people with absolute pitch perceive the pitch of pure tones by using two kinds of strategies. One strategy is that they first place the tones into musical note categories if the tones belong to or are very close to musical notes. In this case, the pitch judgment of absolute pitch possessors remains constant without shifting according to context. This experimental evidence is quite different from the context effect obtained in speech perception, where a strong contrast effect can be obtained. To understand the perceptual mechanisms of auditory events, it is important to know how the context effect occurs in auditory judgments: assimilation, contrast, or no shift. The other strategy is that if it is difficult to categorize the tones into musical notes, subjects use their auditory memory of pitch in the pitch judgment of tones, just as do subjects with nonabsolute pitch.

The present results have important implications for the perceptual strategies of absolute pitch possessors as well as for the time-pitch interdependence observed in the experiments on the auditory judgments.

\section{REFERENCES}

Aвве, M. (1936). The spatial effect upon the perception of time. Journal of Experimental Psychology, 3, 1-52.

BACHEM, A. (1937). Various types of absolute pitch. Journal of the Acoustical Society of America, 9, 146-151.

BORDEN, G. J., \& HARRIS, K. S. (1984). Speech science primer. Baltimore: Williams \& Wilkins.

Burns, E. M., CAMPBELl, S. L., \& Hoberg, K. E. (1989). Is absolute pitch an example of "categorical perception"? In Proceedings of the First International Conference on Music Perception and Cognition (pp. 421-424). Kyoto, Japan.

BURNS, E. M., \& WARD, W. D. (1978). Categorical perceptionphenomenon or epiphenomenon: Evidence from experiments in the perception of melodic musical intervals. Joumal of the Acoustical Society of America, 63, 456-468.

Christensen, I. P., \& Huang, Y. L. (1979). The auditory tau effect and memory for pitch. Perception \& Psychophysics, 26, 489-494.

Cohen, J., Christensen, I. P., \& ONo, A. (1974). Influence of temporal intervals on comparative judgments of pitch: A study of subjective relativity. Tohoku Psychological Folia, 33, 76-87.

Cohen, J., Hansel, C. E. M., \& Sylvester, J. D. (1953). A new phenomenon in time judgment. Nature, 172, 901.

Cohen, J., Hansel, C. E. M., \& Sylvester, J. D. (1954). Interdependence of temporal auditory judgments. Nature, 174, 642.

Cutting, J. E., \& Rosner, B. S. (1974). Categories and boundaries in speech and music. Perception \& Psychophysics, 16, 564-570.

Hesson, H. (1930). The tau effect-An example of psychological relativity. Science, 71, 536-537.

Liberman, A. M., Cooper, F. S., Shankweiler, D. P., \& StuddertKennedy, M. (1967). Perception of the speech code. Psychological Review, 74, 431-461.

MIYAZAKI, K. (1988). Musical pitch identification by absolute pitch possessors. Perception \& Psychophysics, 44, 501-512.

Parks, T. E. (1968). The spatial separation of two light-flashes and their perceived separation in time. American Journal of Psychology, 81, 92-98. 
Pisoni, D. B. (1977). Identification and discrimination of the relative onset time of two component tones: Implication for voicing perception in stops. Journal of the Acoustical Society of America, 61, 1352-1361.

Pisoni, D. B., Carrell, T. D., \& Gans, S. J. (1983). Perception of the duration of rapid spectrum changes in speech and nonspeech signals. Perception \& Psychophysics, 34, 314-322.

Price-Williams, D. R. (1954). The kappa-effect. Nature, 173, 363-364.

Radocy, R. E., \& Boy LE, J.D. (1979). Psychological foundations of musical behavior. Springfield, IL: Charles C. Thomas.

Repp, B. H., Healy, A. F., Crowder, R. G. (1979). Categories and context in the perception of isolated steady-state vowels. Journal of Experimental Psychology: Human Perception \& Performance, 5 , 129-145.

Shigeno, S. (1986). The auditory tau and kappa effects for speech and nonspeech stimuli. Perception \& Psychophysics, 40, 9-19.

SHIGENo, S. (1987). The auditory tau and kappa effects for voiced stop consonants. Japanese Psychological Research, 29, 71-80.

ShIGENo, S. (1991). Assimilation and contrast in the phonetic perception of vowels. Journal of the Acoustical Society of America, 90, 103-111.
Shigeno, S., \& Fujisaki, H. (1979). Effect of a preceding anchor upon the categorical judgment of speech and non-speech stimuli. Japanese Psychological Research, 21, 165-173.

Shigeno, S., Fujisaki, H. (1980). A comparison of forward versus backward context effect in phonetic and non-phonetic judgments on synthetic vowels. Japanese Journal of Psychology, 51, 287-290. (In Japanese, with English summary)

SIEGEL, J. A. (1974). Sensory and verbal coding strategies in subjects with absolute pitch. Journal of Experimental Psychology, 103, 37-44.

Siegel, J. A., Siegel, W. (1977a). Absolute identification of notes and intervals by musicians. Perception \& Psychophysics, 21, 143-152.

Siegel, J. A., \& SiEgel, W. (1977b). Categorical perception of tonal intervals: Musicians can't tell sharp from flat. Perception \& Psychophysics, 21, 399-407.

WARD, W. D., \& BurNs, E. M. (1982). Absolute pitch. In D. Deutsch (Ed.), The psychology of music (pp. 431-451). New York: Academic Press.

(Manuscript received March 16, 1992; revision accepted for publication April 6, 1993.) 